\title{
Fuzzy Logic Based Trajectory Tracking Controller
}

\author{
S.Ravi kumar, R.P Das, A.Sampath Dakshina Murthy, K.Sunil kumar
}

\begin{abstract}
-shape autopilot of ship has been proposed based totally on fluffy reason. A non-direct numerical version for oil tanker is taken for assessment of parameters which shift with profundity. The presentation of proposed controller has been tested as for course by way of the usage of normal AI fluffy framework just as ANFIS

ANFIS based framework for designing autopilot of deliver has been proposed. A nonlinear medical model of an oil tanker is taken for assessment of parameters which shift with profundity. The presentation of proposed controller is to be tried for both converting and direction preserving mode
\end{abstract}

Index Terms- Autopilot, controller, Fuzzy logic, ship, Trajectory Tracking

\section{INTRODUCTION}

Mechanics of photo of ship constant non direct and shift with running situations, as an example, profundity of water and pace simply as outer, erratic additives like ocean flows and wind produced waves and circulation of winds. Nonlinear manage plans, for instance, yield complaint control, back venturing had been accounted for $[1,2,3]$. utilization of valid techniques are identified with the minute to be directed and again joined with hydrodynamics, precise model of marine car is difficult to determine. To defeat such troubles, fluffy cause and neural systems are helpful. Fluffy cause primarily based controllers have been advocated with the aid of severa scientists inclusive of Yang, et.al, Santos, et.al, Velagic, et.al[4,5,6]who proposed deliver autopilot for following via sugeno type fluffy framework Wu,y,et.al consolidated neural device and fluffy cause. essentially Di.move, et.al moreover displayed blend of both.

A large portion of the paintings mentioned did not receive profundity of water as a element furthermore, they proposed fluffy reason coordinated with exclusive systems. it is the purpose of this proposed to build up a fluffy motive controller working sturdy for numerous profundity of region [7].

\section{Deliver factors}

Mobility of marine automobiles is depicted via the factors which consist of both kinematics and energy. Kinematics are identified with precise pace wherein as electricity are identified with reaction while basic amounts are applied. The six degrees of opportunity are flood, influence, hurl, yaw, roll and pitch. The preliminary 3 directions and their time subsidiaries deliver the location and translational movement in $\mathrm{x}, \mathrm{y}, \mathrm{z}$ hub. the second one association of three guidelines are identified with direction and rotational moment of the marine vehicle

\section{Model of Tanker}

$\mathrm{x}=\mathrm{A} \underline{\mathrm{x}}+\mathrm{Bu} \quad \mathrm{x}=[\mathrm{u}, \mathrm{v}, \mathrm{r}]$ is a state vector $\mathrm{A}$ is the system matrix, $B$ is the input matrix and $u=[\delta r]$ is input component (rudder deflection)

\section{Fuzzy Logic Controllers (FLC)}

It should have proper values for any reference angles from $\pm 3^{0}$ to $\pm 45^{0}$. It should give agreeable outcomes for predefined direction by giving arrangement of heading points. The ship should follow the ideal heading with great exactness. Increasingly finished, the distinction between wanted heading and real heading ought not surpass \pm 30 . The control sign ought not achieve the greatest estimation of $\pm 350$

\section{Inputs and outputs of control mechanism}

States influenced by rudder minute which is identified with yaw and influence velocity.The wanted states are takesfrom second subsidiary and the fixed premix is to decrease the mistake [difference among wanted and real states].

Inputs: 1) Heading angle error ( $\psi_{-}$error).

2) Heading state error (r_error).

Output: Rudder deflection ( $\delta \mathrm{r})$

Selection of ranges

Table 1. Universal sets for ranges of input

\begin{tabular}{|c|c|}
\hline Input & Range \\
\hline Heading_error & $-3^{0}$ to $+3^{0}$ \\
\hline
\end{tabular}

Table 2. Output limits

\begin{tabular}{|c|c|}
\hline Output & Range \\
\hline Rudder deflection & $-35^{0}$ to $+35^{0}$ \\
\hline
\end{tabular}

Starting from these values of range of $\mathrm{I} / \mathrm{O}$ variables are obtained by trail and error (experience).

-ve to $+v e=>$ clock wise and anti clock wise $=>$ port to star board.

Universal sets of ranges of $\mathrm{I} / \mathrm{O}$ variables

$\mathrm{I} / \mathrm{P}$ Heading error ( $\psi$ _error) $\rightarrow-0.4^{0}$ to $+0.4^{0}$

Heading rate error (r_error) $\rightarrow-0.01^{0}$ to $+0.01^{0}$

$\mathrm{O} / \mathrm{P}$ Rudder deflection $\delta \mathrm{r} \rightarrow-0.8^{0}$ to $+0.8^{0}$

A Type of Membership Function

Revised Manuscript Received on April 12, 2019.

S.Ravi kumar, India.

Dr.R.P Das, India.

A.Sampath Dakshina Murthy, India.

K.Sunil kumar, India. 


$$
\mu\left(x_{\text {error }}, a, b, c\right)=\left\{\begin{array}{cc}
0 & x_{\text {error }}<a, x_{\text {error }}>c \\
\frac{\mathrm{x}_{\text {error }}-\mathrm{a}}{\mathrm{b}-\mathrm{a}} & \mathrm{a} \leq \mathrm{x}_{\text {error }} \geq \mathrm{b} \\
\frac{\mathrm{c}-\mathrm{x}_{\text {error }}}{\mathrm{c}-\mathrm{b}} & \mathrm{b} \leq \mathrm{x}_{\text {error }} \geq \mathrm{c}
\end{array}\right.
$$

Subsets BN-Big negative MN-Medium negative ZE-Zero SP-Small positive MP-Medium positive BP-Big positive

\section{Fuzzy variables and subsets}

Heading error as a range -0.533 to +0.530

Heading rate error as a range -0.13330 to +0.13330

Rudder rate as a range -1.067 to +1.670

\section{IF THEN Rules}

1)IF $\rightarrow$ Antecedent THEN $\rightarrow$ Subsequent

The no.of rules depends on number of inputs,no.of subsets/membership functions [10] and number of output

2) seven subsets $7 X 7=49$ rules

Some of the rules are[7,8]

1) IF heading rate error is BN AND heading error is BN THEN rudder is $\mathrm{BN}$

2) IF heading rate error is MN AND heading error is MN THEN rudder is $\mathrm{MN}$

3) IF heading rate error is SN AND heading error is SN THEN rudder is $\mathrm{SN}$

4) IF heading rate error is ZE AND heading error is ZE THEN rudder is ZE

5) IF heading rate error is SP AND heading error is SP THEN rudder is SP

6) IF heading rate error is MP AND heading error is MP THEN rudder is MP

and so on

Defuzzification \& RESULTS

$$
\mathrm{u}=\frac{/ \mu(z) z d z}{\sqrt{\mu}(z) d z}
$$

ANFIS is a combination of a neural networks and fuzzy logic in which fuzzy factors are adoptive. Neural networks have learning algorithms where as fuzzy system generates signals for controlling is through fixing of rule base this rule based is taken from previous data and is random the implication is that the output of the controller is also random which can present optimum results.

ANFIS makes the guidelines primarily based completely choice more adaptive to the state of affairs which utilises the Neural Networks through decrease back propagation for reinforcing the overall performance. The residences of fuzzy good judgment in approximating a non-linear system via if then hints is used in the modelling. In ANFIS crisp signs are converted into fuzzy inputs thru membership features and these are fed to neural network block .

Back Propagation is used for neural community in right selection of rule based definitely. as quickly as education is finished right tips may be generated and hired via NN to feed most dependable output. Linguistics output of $\mathrm{NN}$ is converted to crisp output through defuzzification. The nodal outputs are combined to the crisp outputs that is in comparison with favored price and errors is comments precept of ANFIS
In ANFIS(Adaptive Neuro-Fuzzy inference system) policies are developed all through education this is information primarily based absolutely FIS (Fuzzy inference gadget) has membership derived from schooling examples. There are forms of FIS and Sugeno. In Mamdani type, output membership is triangular or and sugeno is linear or consistent. Sugeno is pc powerful where asmamdani is much less effective depending on expert information.

\section{Architecture}

\section{If input are $\mathrm{X}$ and $\mathrm{Y}$}

Rule 1: If $X=A 1, Y=B 1$ then $f 1=p 1 X+q 1 Y+r 1$

Rule 2: If $X=A 2, Y=B 2$ then $f 2=p 2 X+q 2 Y+r 2$

Here A1 and B1 are Fuzzy sets, $\mathrm{f} 1$ is the output. Design parameters determined by training process $\mathrm{p}, \mathrm{q}, \mathrm{r}$

\section{Layers}

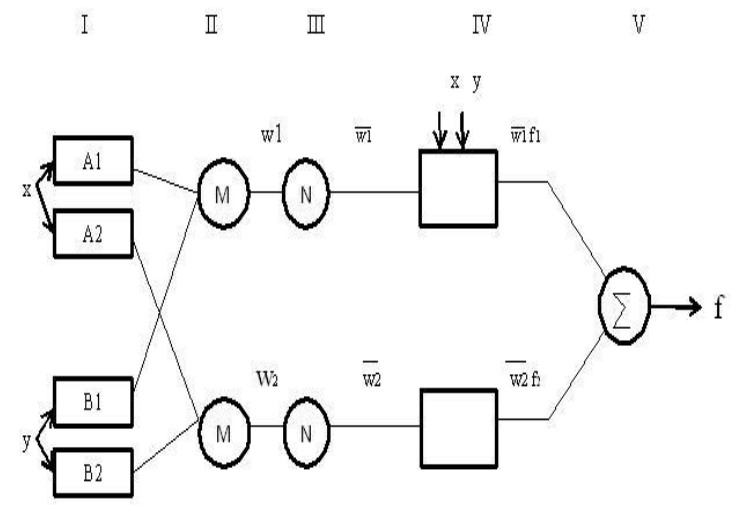

Fig.1. Layered structure of ANFIS

$\mathrm{O}_{\mathrm{i}}^{\mathrm{j}} \mathrm{o} / \mathrm{p}$ of $\mathrm{i}^{\text {th }}$ node, $\mathrm{j}^{\text {th }}$ layer

Layer 1: $\mathrm{Oi}^{1}=\mu \mathrm{Ai}(\mathrm{X})$

$\mathrm{i}=1,2$

Ai is linguistic label such as cold hot

Layer 2: Each node determines firing strength of a rule by multiplicative

$\mathrm{Oi}^{2}=\mathrm{w} 2=\mu \mathrm{Ai}(\mathrm{X}) \mathrm{x} \mu \mathrm{Ai}(\mathrm{Y})$

$\mathrm{i}=1,2$

Layer 3: Firing strengths are normalized to distinguish between the firing strength strengths of each rule for the total furry strength of total rules.

$$
\mathrm{Oi}^{3}=\overline{w 1}=\mathrm{wi} /(\mathrm{w} 1+\mathrm{w} 2) \quad \mathrm{i}=1,2
$$

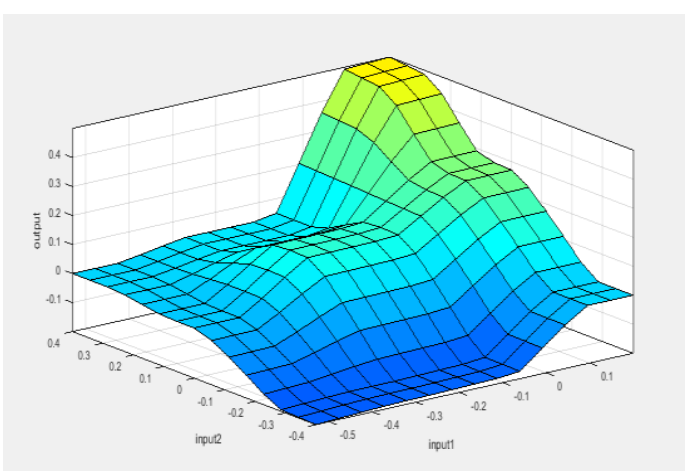

Fig.2. 3D Surface view of ANFIS 
Input 1 as heading rate error and input 2 as heading error output as rudder deflection

ANFIS info: WITH NORMAL MODE

nodes: 35

linear parameters: 27

nonlinear parameters: 24

Total parameters: 51

training data pairs: 7

checking data pairs: 0

fuzzy rules: 9

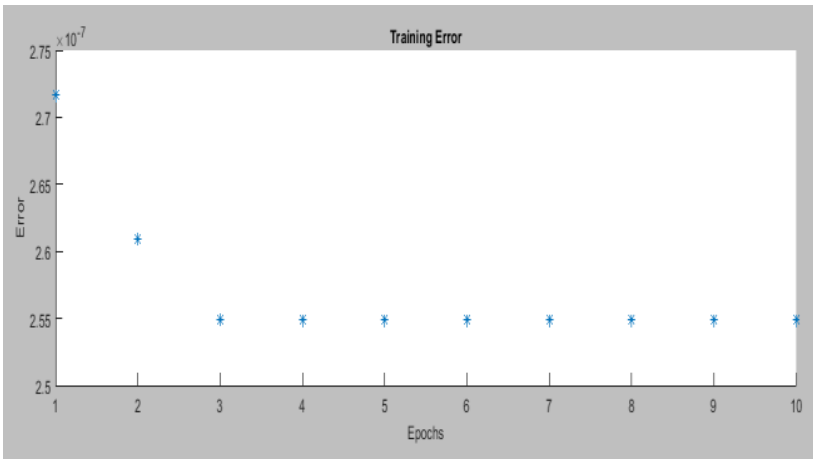

Fig.3.Training error for 10 epochs

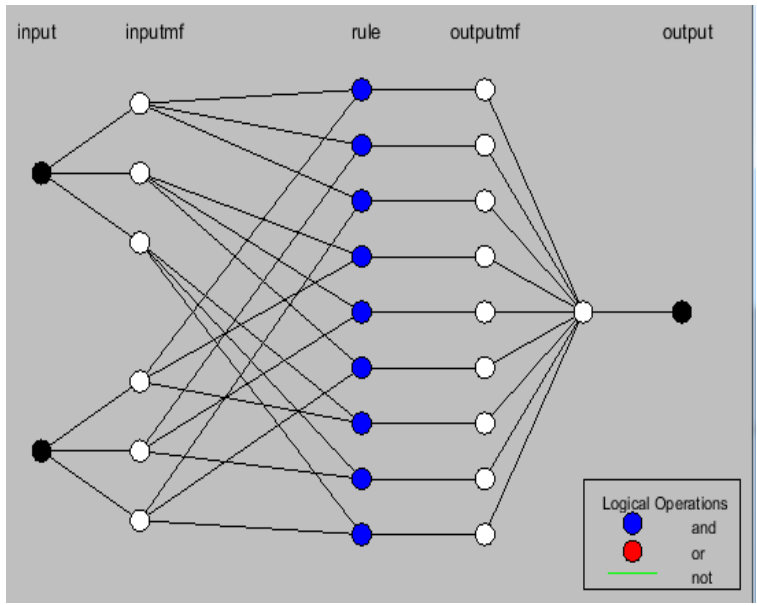

Fig.4.Structure of ANFIS

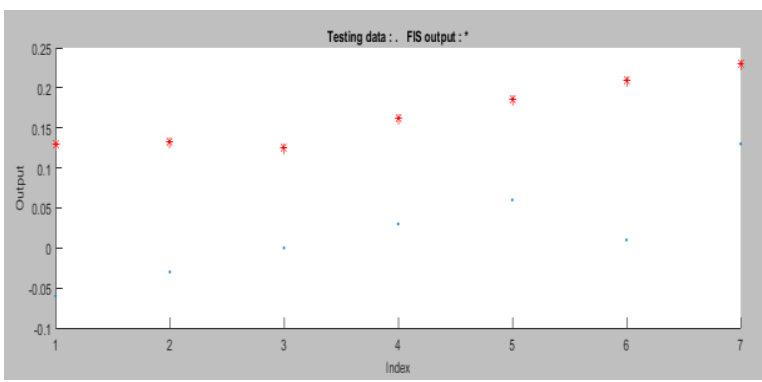

Fig.5. Anfis Using Fuzzy Output

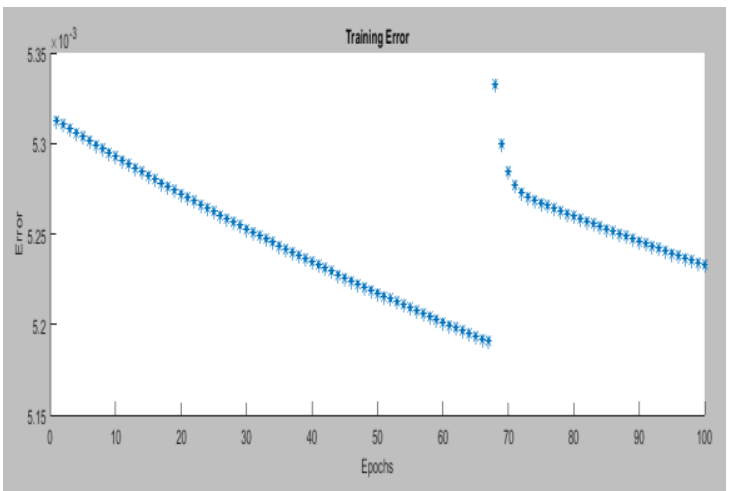

Fig.6.Training error for 100 epochs

ANFIS info: with RMSE USING BACK PROPAGATION METHOD

nodes: 35

linear parameters: 27

nonlinear parameters: 24

Total parameters: 51

training data pairs: 7

checking data pairs: 7

fuzzy rules: 9

Minimal training RMSE $=0.001022$

Minimal checking RMSE $=0.00102177$

Epoch 100 error $=0.00523$

Final outputs

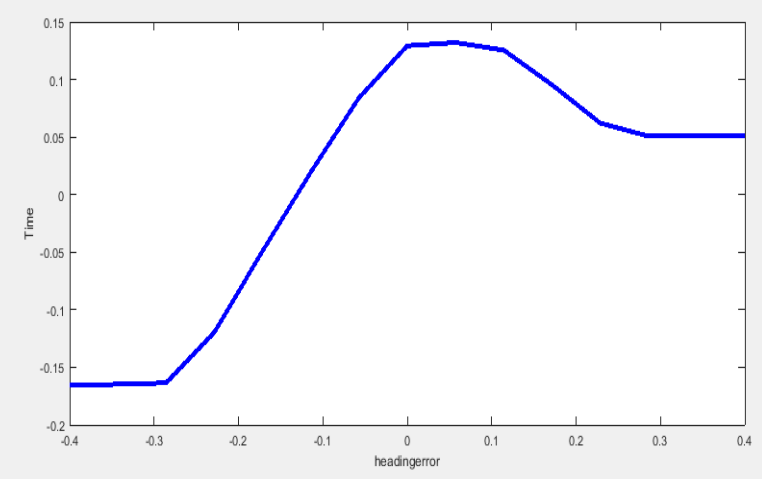

Fig.7.Heading error

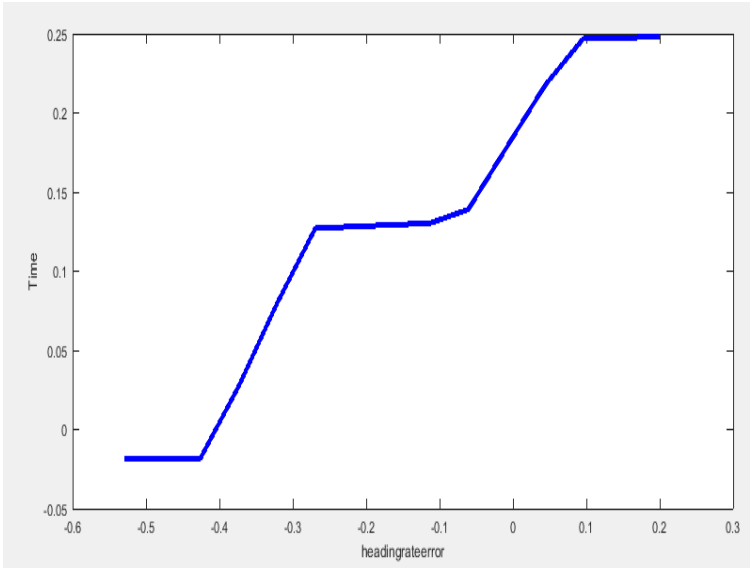

Fig.8.Heading_rate error

Published By:

Blue Eyes Intelligence Engineering

\& Sciences Publication 
TABLE 3. Comparision between AI, ANFIS, RMSE

\begin{tabular}{|l|l|l|l|}
\hline Method & AI & ANFIS & RMSE \\
\hline $\begin{array}{l}\text { Back } \\
\text { propagation }\end{array}$ & 0.01 & 0.025 & $2.54943 \mathrm{e}-07$ \\
\hline Hybrid & 0.09 & $\begin{array}{l}0.0001 \\
100\end{array}$ & $9.19762 \mathrm{e}-07$ \\
& 100 & $\begin{array}{l}\text { Epoch } \\
\text { Epoch }\end{array}$ & \\
\hline & 0.093 & 0.003077 & 0.00307744 \\
& 200 & $\begin{array}{l}200 \\
\text { Epoch }\end{array}$ & \\
& Epoch & Eoch & \\
&
\end{tabular}

\section{CONCLUSION:}

The conventional FIS is an ANFIS are the guideline systems which can be proposed and thought about for settling on a green technique for format og autopilot. the capacity of ANFIS is obvious to be the fundamental motivation behind inclination while effortlessness is the power of the customary fluffy method

\section{REFERENCES}

1. Fang, Y., Zergeroglu, E., deQueiroz, M., and Dawson,D.M., "worldwide Output remarks manipulate of Dynamically Poitioned floor Vessel: An Adaptive manage method", Mechatronics, volume 14, pp. 341-356,2004.

2. Zhang, R., Chen, Y., solar, Z., sun, F., and Xu, H., "manner manage of floor ship in confined Waters the usage of Sliding Mode", IEEE Transaction on manage gadget generation, extent eight, No. four, pp. 722-732, 2000

3. Do, okay.D., Jiang, Z.P., and Pan, J., "lively Adaptive direction Following of Underactuated Ships", Automatica, quantity forty, pp. 929-944, 2004.

4. Yang, Y., and Zhou, C., "versatile Fuzzy manage of deliver Autopilot with unsure Nonlinear systems", complaints of IEEE convention on Cybernetics and wise systems, Singapore, 2004.

5. Yang, Y., "Direct sturdy Adaptive Fuzzy manipulate(DRAFC) for uncertain Nonlinear systems using Small benefit Theorm", Fuzzy sets and systems, extent 151,pp. 79-ninety seven, 2005.

6. Velagic, J., Vukic, Z., and Omerdic, E., "flexible Fuzzy supply Autopilot for song maintaining," manage Engineering exercise, volume.eleven, pp. 433-443, 2005.

7. Pathan, D.M., Unar, M.A. furthermore, Memon, Z.A., 2016. "Fluffy intent route following controller for a tanker". Mehran university studies magazine of Engineering and generation, Vol. 31, No. 2, April 2012

8. Das.R.P ,L.Sreedhar "Neural systems and fluffy cause" 